\title{
Magnetic Topology before a 3B/X1.2 Flare and the Associated CME
}

\author{
H. $\mathbf{L i}^{1}$, B. Schmieder ${ }^{2}$, G. Aulanier ${ }^{2}$ and A. Berlicki ${ }^{2,3}$ \\ ${ }^{1}$ Purple Mountain Observatory, Nanjing 210008, China Email: lihui@mail.pmo.ac.cn \\ ${ }^{2}$ Observatoire de Paris, Section de Meudon, LESIA, F-92195, Meudon Principal Cedex, France \\ ${ }^{3}$ Astronomy Institute of the Wrocław University, ul.Kopernika 11, 51-622 Wrocław, Poland
}

\begin{abstract}
We extrapolated observed photospheric magnetic field before the 3B/X1.2 flare occurred in NOAA 10486 on 2003 October 26. Two magnetic null points exist above the active region, which have no crucial role in triggering the flare, even though enhanced TRACE EUV/UV emission was observed before the main flare due to magnetic reconnection near the lower-altitude null point. We conclude that this flare results from the loss of equilibrium due to persistent flux emergence and photospheric motion, and strong shear. The accompanying fast CME with velocity of $\sim 800 \mathrm{~km} \mathrm{~s}^{-1}$ shows quick mass pickup and energy increase in the low corona. Its kinetic energy is always larger than its potential energy.
\end{abstract}

Keywords. sun: flares, sun: magnetic field, sun: UV radiation.

\section{Introduction and Observation}

Solar flare and coronal mass ejection (CME) are the most energetic activity in the solar atmosphere, and closely related to the magnetic field in the associated active region. Complicate magnetic topology and strong magnetic shear are demonstrated to be critical for a flare and CME to occur. Parametric evolutions of CMEs will shine some light on better understanding the physics of CMEs.

Data used in the paper are from a number of ground-based and space-borne instruments, including the Fench-Italian telescope THEMIS at Canary islands, TRACE, $\mathrm{SOHO} / \mathrm{MDI}, \mathrm{SOHO} / \mathrm{LASCO}$.

\section{Results}

By assuming a current-free field configuration $(\vec{\nabla} \times \vec{B}=0)$ and using the code developed by Démoulin et al. (1997), we extrapolated the photospheric magnetic field obtained by THEMIS at 08:56 UT after inserting into large MDI magnetogram at 07:15:03 UT to infer the large scale three dimensional (3D) magnetic structure above active region NOAA 10486 before the 3B/X1.2 flare on 2003 October 26. The results clearly demonstrate the existence of two magnetic null points above the region. One (called NP1) is rather high in the corona $(\sim 7.0 \mathrm{Mm})$ and another (called NP2) is just in the transition region $(\sim 2.25 \mathrm{Mm})$ (Fig. 1(b)). The fan and spine structures of NP2 intersect with the photosphere in A, C, D, and G, while NP1 in A, B, F, and G.

There were already some bright patches in TRACE $1600 \AA$ images 7 min before the X-ray flare onset (regions A, C, D, E, G in Fig. 1(a)). We computed the light-curves of thee 8 regions shown in Fig. 1(a), which clearly show enhanced emission in four regions (A, C, D \& E) around 05:50 UT in both $1600 \AA$ and $195 \AA$, implying the atmosphere there was heated to some extent, i.e., small amount of energy released around 05:50 

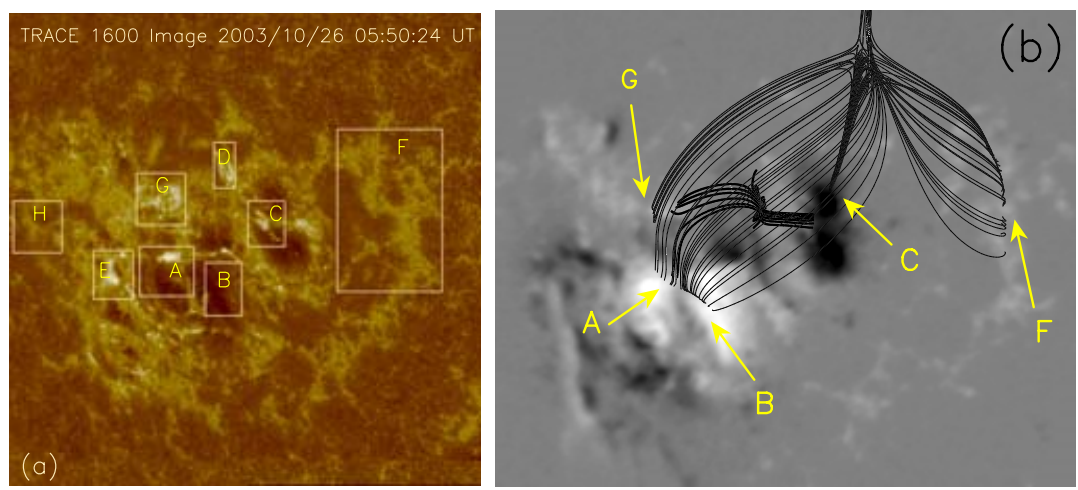

Figure 1. (a) TRACE $1600 \AA$ image at 05:50:24 UT; (b) top view of the extrapolated magnetic field lines using the longitudinal magnetic field observed by THEMIS at 08:55:52 UT.
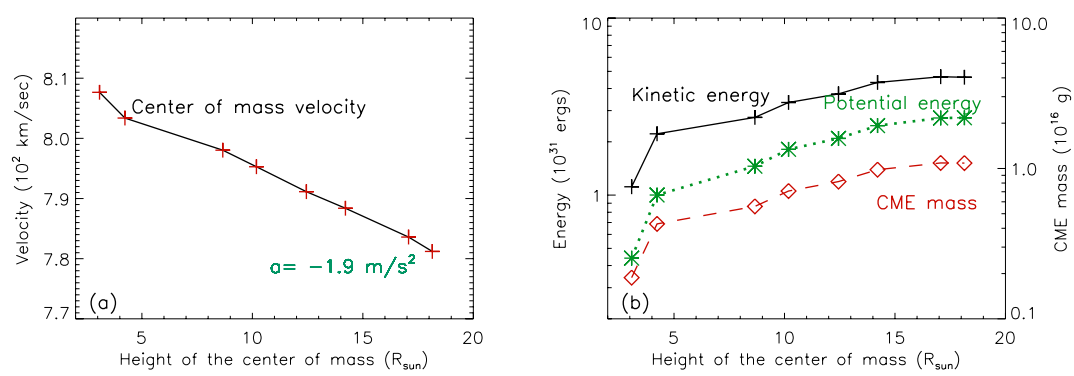

Figure 2. Variations of CME parameters with height of the center of mass: (a) center of mass velocity, (b) kinetic (solid) and potential (dotted) energy, and mass (dashed).

UT. However, the enhanced emissions are only related to NP2 and have nothing to do with NP1. Therefore, the existence of magnetic null point is not a sufficient condition to trigger this eruptive flare. This flare could result from the loss of equilibrium due to persistent flux emergence, continuous photospheric motion, and strong shear.

A fast CME associated with the 3B/X1.2 was first seen by LASCO/C2 at 06:54 UT. We use the method of Vourlidas et al. (2000) to compute the evolution of its mass, center of mass velocity, acceleration, kinetic and potential energy. The results are shown in Fig. 2. The velocity was calculated by a second order fitting to the height-time curve of the center of mass, which yields a slowly decreasing velocity of about $800 \mathrm{~km} \mathrm{~s}^{-1}$ $\left(a=-1.9 \mathrm{~m} / \mathrm{s}^{2}\right)$. The figure shows that the mass and energy of the CME increase quickly in the low corona, and tend to be constant in the outer corona above about 15 solar radii. The kinetic energy $\left(E_{k}\right)$ is always larger than the potential energy $\left(E_{p}\right)$, and their ratio $\left(E_{k} / E_{p}\right)$ decreases from 2.5 in the low corona to 1.7 in the outer corona.

\section{Acknowledgements}

This work was partial supported by the National Natural Science Foundation of China (NSFC grant No. 10573038 \& 10333040) and the European Commission through the RTN programme (HPRN-CT-2002-00313).

\section{References}

Démoulin, P., Bagala, L.G., Mandrini, C.H. et al. 1997, Astron. Astrophys. 325, 305

Vourlidas, A., Subramanian, P., Dere, K.P. \& Howard, R.A. 2000, ApJ 534, 456 